\title{
Social Media Use of Older Adults: A Mini-Review
}

\author{
Anja K. Leist \\ Faculty of Language and Literature, Humanities, Arts and Education, University of Luxembourg, \\ Walferdange, Luxembourg; Department of Public Health, Erasmus Medical Center, Rotterdam, The Netherlands
}

\section{Key Words}

Social contacts · Technology · Loneliness · Old age · Older people $\cdot$ Social media . Online social networks . Online communities

\begin{abstract}
Background: Maintaining social relationships has been defined as a core element of aging well. With a considerable amount of older adults living alone, social media provides the possibility to engage in meaningful social contact, e.g. by joining online social networks and online discussion forums. Objective: The review encompasses current knowledge of prerequisites in social media use of older adults such as functional capacity, information and communications technology-related knowledge, and favorable attitudes towards social media. Then, the potential of social media use for clinical practice and possible negative consequences are outlined. Method: Literature on social media use from a gerontological perspective was reviewed in July and August 2012. Results: Online communities are suitable for providing and receiving social support when confronted with a difficult life situation, regardless of geographical location or time. From a practitioner's perspective, social media can be used to advance health-related knowledge such as informa-
\end{abstract}

tion on prevention, diagnosis, and treatment of specific conditions and disorders. Further positive consequences have been shown to be overcoming loneliness, relieving stress, and raising feelings of control and self-efficacy. Possible negative consequences could be misuse of personal data as well as the distribution and uncritical adoption of potentially harmful information via online communities. Discussion: The potential of social media in clinical practice is reflected in a wide range of intervention possibilities for older adults. However, with the rise of social media, new threats emerge for older adults as well.

Copyright $\odot 2013$ S. Karger AG, Basel

\section{Introduction}

Maintaining meaningful social relationships is widely regarded as one of the key elements of aging well. Geographical distance to kin, impaired mobility or time-consuming obligations such as caregiving may hinder older adults from satisfying the need for social contact, with the risk that these adults feel lonely, but have little opportunity to engage in social contact. Social media may overcome these barriers as online social networks and online discussion forums can be used to engage in social

\section{KARGER}

(C) 2013 S. Karger AG, Basel

0304-324X/13/0594-0378\$38.00/0

E-Mail karger@karger.com

www.karger.com/ger
Dr. Anja K. Leist

Faculty of Language and Literature, Humanities, Arts and Education

University of Luxembourg, Route de Diekirch, B.P.2

LU-7201 Walferdange (Luxembourg)

E-Mail anja.leist@uni.lu 
contact regardless of geographical location or time. Moreover, social media provides new possibilities to engage in social contact, to provide and receive support, and to raise feelings of control. A number of research projects are currently being undertaken using information and communications technology (ICT) to facilitate older adults' participation in online social networking and online communities. An example is the project TAO (Third Age Online), which is funded under the second call of the European Ambient Assisted Living Joint Programme, which aimed at funding research for new technologies to socially connect older adults [1]. With an increasing number of older adults using the Internet, social media use among older adults is continuously spreading. As of April 2012, half of the adults aged 65 years and older in the USA are now online, and every third online senior (34\%) uses social networking sites like Facebook and LinkedIn [2]. Social media applications are also considered additional helpful features in telehealth and telecare services [3].

Social media in general relies on computer-mediated communication and is defined as tools and platforms with the goal to consume, co-create, share, and modify user-generated content. Furthermore, these applications can be used to interact with other users via collaborative projects, blogs, content communities, social networking sites, virtual game worlds or social worlds [4]. A related concept is Web 2.0, which describes web content as being created and modified by a range of users. Social network sites are web-based services where individuals can display a public profile and a list of other users with whom they share a connection, as well as search profiles and lists of other users.

Participation in social media can range from quite passive behavior such as reading posts and online discussions (often called lurking) up to active participation by posting, blogging or uploading multimedia content. It is possible to address a broad audience of community members with posts or messages, but also single persons or subgroups can be addressed. Privacy of these messages varies depending on the network, the application under use (e.g. personal message, restricted discussion board, open forum), and the user alias (e.g. nickname or real name). The amount of shared personal information ranges from superficial information such as chitchatting and recommending ('liking') web content up to disclosing conditions and disorders or other personal experiences. Online communities like SeniorNet [5], online social networks, and other platforms that are specifically addressing older users are growing steadily.
Considering the novelty of the concept of social media and the manifold intervention possibilities for older adults, it seems timely to review social media use in older age from a gerontological perspective. A literature search was carried out in July and August 2012 (the full literature list can be requested from the author). With the aim of giving a broad overview of existing knowledge and gaps in social media use of older adults, this contribution addresses prerequisites in social media use, its potential in clinical practice, and possible negative consequences of social media use.

\section{Prerequisites in Social Media Use}

Important prerequisites in social media use are sufficient functional capacity to use a computer or a web-enabled device in a meaningful way as well as the adoption of ICT-related knowledge in general and of social media in particular. Then, attitudes towards and motivations for social media use will be presented. Last, by identifying shortcomings of older adults' current social media use, ways to overcome barriers will be depicted.

\section{Functional Capacity}

A considerable number of older adults suffer from functional limitations, such as limited cognitive and perceptual abilities [6], which hinder them from using technology in a comparable way to younger adults. Limitations like blindness may, despite assistive technology, even prevent the use of online social networks if no further assistance is available.

Further, despite objectively sufficient capacity, attitudes and beliefs on insufficient functional capacity have been found to constrain ICT use of older adults [6]. Investigating general ICT acceptance in older age often reveals computer anxiety and general negative views towards ICT use. Lee et al. [7] identified different kinds of perceived barriers, such as intrapersonal (e.g. 'I am too old for it'), functional (e.g. related to memory decline, spatial orientation), structural (e.g. 'cost too much to own a computer'), and interpersonal (e.g. 'no one teaches me how') barriers. In general, barriers were rated less constraining with higher income and higher education, but perceived as more constraining with increasing age of the users [7]. These perceived barriers make older adults a population that might not as easily adopt and use ICT applications and social media as younger generations. 


\section{Adoption of ICT- and Social Media-Related Knowledge}

An important prerequisite of ICT use in general is a certain web proficiency; concepts like browser, server, search engine, etc., need to be adopted in order to understand the process of searching and accessing websites and applications. On top of these general concepts, additional knowledge is necessary for using social media. First, terms and rights of online social networks and websites where personal and other information is stored should be understood, as well as knowledge on how the company behind the online social network uses personal data and if it distributes these data to other companies [8]. Second, application-specific knowledge on functionalities to engage in consuming, creating and sharing social media content is necessary. Social media use of older adults has, to date, mainly been investigated as participating in online discussions and instant messaging; only few older adults engage in actually creating content such as uploading pictures or videos. It is expected with the availability of user-friendly mobile applications (e.g. on tablets or smartphones) that social media will be used more readily by older adults in the next years.

\section{Attitudes toward Social Media Use}

Most of the reviewed studies focused on novice participants and followed them over a limited period of time, so that mainly the reception, and to a lesser extent the process of sharing and co-creating user-generated content, has been investigated. Upon first encounter, reception of social media such as online social networks is quite negative for several reasons. First of all, some online social networks have received media attention about problems with data privacy protection, which resulted in negative attitudes towards the 'brand' of a social network. This may prevent older adults from joining these networks [8]. A second reason for negative perceptions is that novices perceive a lack of code of social conduct in online communities and are thus reluctant to participate in these communities. This is due to the fact that online social norms differ from offline social contact. In offline contexts, behavior is quite formal except between friends, kin, and close acquaintances, whereas in the online context, most users apply informal behavior to communicate with other community members.

Third, novice users notice that forms of self-disclosure and self-representation are different in offline and online social behavior. Offline behavior is expected to be modest and polite. However, in online social networks, it is widely acceptable to upload pictures of oneself, 'broadcast' achievements, and generally present oneself in a favorable light. Consequently, material posted in online social networks is perceived by novices as self-centered and embarrassing, and even as 'silly and empty-headed' [9]. However, studies also show that after a period of time where social media use was encouraged and benefits were highlighted, study participants continuously engaged in social media and reported positive consequences afterwards. A fourth reason for negative attitudes toward social media is pointed out by Xie et al. [8], who found that novices experience a lack of personal relevance in social networks. This finding, though, may be due to the fact that personal relevance of joining an online community or an online social network had not been established before, such as choosing networks where family members or friends are already participating. For continuous engagement in social media it is necessary that the user establishes personal relevance. Thus, it may be advantageous for novice users to search for specific web content and online communities that are in some way linked to hobbies, interests or needs rather than to join a large online social network with a broader audience.

Last, novice users often perceive a lack of control in managing registration and fear that data privacy may be violated $[6,8]$. In line with this, Pfeil et al. [10] note that uncertainty about the identity and intentions of other users may lead to a reluctance to join online communities and participate in social media. These attitudes are certainly not restricted to older generations. However, younger adults seem to adopt strategies to deal with these issues, such as modifying privacy settings, regularly deleting their online content, or restricting themselves to sharing only innocuous information. In contrast, the literature suggests that older novice users often do not feel capable of dealing with these issues and are reluctant to join online social networks. However, indirect evidence for the general population of older adults having more positive attitudes than the reviewed studies is suggested from user numbers [2]: the US educational site SeniorNet currently receives over 1 million hits per month [5]. Qualitative analyses of senior online communities make use of extensive web content, e.g. in a study by Nimrod [11], indicating active participation of a high number of senior users.

To conclude, attitudes towards social media have mainly been investigated with novice users and with a focus on large online social networks, finding that novices often perceive a lack of personal relevance and miss a code of conduct in these informal networks. However, 
hits on and content of websites aiming at older users provide indirect evidence for positive attitudes of more Internet-savvy older adults compared to the novice study participants.

\section{Motivations for Social Media Use}

The main motivations for older adults to use social media are enjoyment, engaging in social contact, and to provide and receive social support. Especially if users engage in online social networking continuously, they are motivated by feelings of enjoyment during use. Similarly, in content analyses of online communities of older adults, the most important topic has been identified as 'fun' [11]. Enjoyment can be enhanced by integrating games and other distractions into applications, but should be regarded mainly as the result of an (online) activity that complies with needs and interests of the person.

Second, online communities are identified as places where people can get together and engage in social contact, e.g. overcome loneliness at nighttime and to receive up-to-date information on family members and acquaintances.

Third, social media can be used to provide and receive social support $[12,10]$. Here, users value general characteristics of computer-mediated communication such as anonymous, asynchronous, and text-based communication to connect with others. Social support is especially valued when it comes from people with a similar background or similar experiences, which has been shown for online forums on depression, cancer, alcoholism or caregiving issues. Clearly, the Internet provides manifold possibilities for users with similar background to get in contact, regardless of time and geographical restrictions. However, Lewandowski et al. [13] found that in the face of very adverse critical life events, support primarily given via face-to-face communication was preferred over support via computermediated communication. Also, the amount of information revealed in online social networks may be dependent upon the network characteristics, as with increased number of network 'friends', users may refrain from revealing private information as they perceive social monitoring.

\section{Ways to Overcome Barriers in Using Social Media}

A main barrier is the process of initially entering online social networks or online communities. It is highly recommended that novice users are supported by a mod- erator or an Internet-savvy mentor in order to get acquainted with data privacy protection issues and other relevant information. Also, many online social networks help new members through the registering process, but discussions and sharing content are not actively monitored by a 'visible' moderator. This may be perceived as positive by Internet-savvy members who may from time to time act as moderator themselves, but less savvy users may prefer to have an assigned moderator to whom they can address questions. Also, the moderator may be helpful in establishing and maintaining the 'code of conduct'. A moderator instantly rewarding for active participation may thereby promote continuous engagement of users in the network.

Another barrier refers to the process of accessing web content in user-friendly applications. For novice users, the multiplicity of existing applications and websites is surely overwhelming, extensive menus might be confusing, and a too large set of suggested actions on a website might have the reverse effect of a user taking no action at all. Following this insight, a significant number of current research projects that are designing user-friendly applications are preselecting adequate web content. This can be done by devising the application in a way that only one particular online social network can be accessed, only part of an extensive hierarchical menu is displayed, or the number of links or actions on a website is restricted. The intention is that by preselecting content, older users can access social media applications more easily. However, this strategy bears the risk that the users' options are restricted too much and users cannot decide for themselves which web content they want to access. This is especially undesirable if it is combined with ageist stereotypes that portray older adults as anxious, skeptical, frail, and passive. Also, it is widely neglected that a large number of older adults have moderate and, in some cases, even extensive technological and computer experience. These problems - restricting web content together with ageist stereotypes - pose the threat that user-friendly applications keep older adults in a passive consumer role. For example, one application designed for older adults is an electronic picture frame displaying photographs of the older user's family members, which they had uploaded on Facebook shortly before [14]. This device is promoting passive behavior of the older user, without giving him or her the possibility to reciprocate or comment. To conclude, social mediabased applications should be tailored to users' competencies and needs. Older adults should be assigned an active and creative role and should remain in as much 
control as possible regarding the web content they want to access.

To date, not much is known about effects of differences in design of social media applications for older adults, which may be due to both the novelty of the concept and language barriers. Additionally, there is (1) a lack of systematic knowledge on the effects of user interface design on user acceptance and actual use of social media of older adults, especially with regard to universal design concepts and accessibility issues [15], and (2) a lack of systematic investigations on how social media is perceived in the context of other web content. Recent studies have investigated single national and international social networks and online communities that are intended either to be used by the general population (e.g. Facebook) or specifically by older adults. As there is a wide range of senior online social networks (as some European examples may serve, e.g. seniorentreff.ch, seniorennet.be, senioren.nl, feierabend.de, and quintonic.fr), large differences in purpose, interface, and content exist. To date, no comparative study has evaluated the content and acceptance of different national social networks for older adults. Such a comparative study on existing senior social network sites and other social media applications could certainly advance knowledge about the requirements of new applications, e.g. in the telecare sector.

\section{Potential of Social Media Use in Clinical Practice}

Websites enriched with social media applications such as discussion boards can be used for many clinical purposes. One prominent goal is to address patients or relatives of patients about their condition or disorder using information referring to prevention, diagnosis, and treatment. This information can be given on websites or online forums dedicated to specific illnesses and chronic conditions. Here, social media can be used to distribute relevant information such as health care advice about cancer diagnosis and treatment. Websites can be used to inform about risk factors and about favorable changes in diet or lifestyle in order to cope with the condition. The use of discussion boards may facilitate patient-doctor or patient-nurse communication [16], may strengthen patients' feelings of control, or - by support of other online members - may encourage lifestyle and health behavior changes. Several forms of online social support targeted at patients undergoing orthopedic surgery or caring for adults with intellectual disabilities have been proposed $[16,17]$.
Aside from satisfying the need for social contact, social media can enable users to provide and receive social support, both of which may in turn contribute to life satisfaction and well-being. Here, a clear advantage of social support given online has been identified: in revealing otherwise painful or embarrassing information, self-disclosure is perceived easier via ICT than via eye-to-eye contact. This is called the disinhibition effect [18].

For problem- and topic-oriented online discussion groups, problem-specific exchange, especially with other people with similar conditions or life situations, is highly valued and has been shown to have positive consequences. Studies show positive effects of participation in online social networks and online communities. Number of posts or the time spent in a network have been used as measures of activity; several studies show that benefits are stronger with higher active participation in a network or a forum. A study by Nahm et al. [19] showed that using a discussion board on a hip fracture prevention website enhanced users' learning experience, and gains in hip fracture prevention knowledge were dependent upon the length of use of a discussion board. The amount of active participation in an online forum was influential in relieving stress [20]. In another study investigating an online forum of caregivers, the number of messages posted per week attenuated the negative association between caregiver strain and well-being [21].

Social media can provide older adults with empowerment, a construct that refers to a global sense of connectedness and increased control and self-efficacy [18]. Barak et al. [18] note that feelings of empowerment result from five factors involved in the participation in online support groups, which are (1) psychological impact of writing; (2) expression of and connecting to emotions; (3) gaining information and knowledge; (4) effects of interpersonal relationships and social processes, and (5) influences on decision-making and resulting action. Thus, it seems promising to engage users in online support groups. Similarly, traditional approaches like journaling, which can be used for purposes such as personal development, life documentation and health promotion, can be enriched by using Web 2.0 tools to engage in collaborative projects [22].

Taken together, through information and support exchange in online social networks and discussion boards, life satisfaction, well-being, connectedness as well as empowerment can be enhanced. 


\section{Possible Negative Consequences of Social Media Use}

To date, not much is known about potentially negative consequences of social media use of older adults, and examples provided here are derived from research with adolescents and younger adults. Main drawbacks lie in the potential of adoption of harmful information shared by other users, harmful behavior of other users, and misuse of personal information with criminal intent.

A potential caveat lies in the fact that almost indefinite amounts of information are accessible over the Internet. Doing a web search, e.g. on health-related information, may lead to dozens if not hundreds of relevant websites displaying a vast array of information on changing lifestyle and health behaviors. Such an overload of information may backfire, leading to the perception that the discrepancy between actual and desired lifestyle is too large to succeed in improving lifestyle. This pitfall applies particularly to older users who have been shown to process Internet content more slowly than younger users and may more easily be overwhelmed by too much information.

In online forums where members can share their knowledge and experiences, a threat emerges from sharing and adopting potentially harmful information. Considering the interest of older adults towards health-related information, there is a potential threat if incorrect, ineffective or even harmful information is shared in discussion forums and adopted without reflection, for example layperson advice on medical treatment. Especially in forums where potentially life-threatening chronic conditions and illnesses, like cancer, are discussed, it is highly problematic if potentially harmful content is displayed in a noncritical manner. Therefore, health forums should ideally be monitored and led by a moderator. The review by Luxton et al. [23] may serve as an example of web accessibility of harmful information by showing that the Internet provides anti-suicide information and prevention possibilities, but also a considerable amount of pro-suicide information and other content that may enhance suicide risk. Also, new forms of harmful behavior emerge from engaging in social media, as illustrated in the (extreme) example of cybersuicide, which is a pact between online acquaintances to commit suicide together [23].

Other potential threats of social media use have been identified for children and younger adults, but are currently not well researched for the population of older adults. With the rise of older adults engaging in online social networks and online discussions, these phenomena may pose a threat to older users as well. Due to positive self-portrayal and sharing mainly positive information in social networks, users who spend a lot of time in online social networks perceive other users as happier and having better lives compared to themselves [24]. Today, mainly younger adults become victims of a harmful behavior called cyberbullying, meaning that posts or messages are published online with the intention to hurt or embarrass another person. With increasing use of social media, older adults may become victims of cyberbullying as well. However, older adults may differ from adolescents in terms of stability of self-concept and existence of lifetime social bonds. Furthermore, for adolescents, online social relationships may more directly reflect their offline social relationships, whereas this may not necessarily be the case for older adults. To conclude, to what extent older adults may suffer from cyberbullying and how to address this problem should be the subject of future research.

Due to limited knowledge of appropriate Internet disclosure, older adults may reveal too much personal information that can be misused. An extreme example of misuse of personal data is identity theft. Further, uploading holiday pictures after providing information on the home address could lead to robbery of one's home. Public media have drawn attention to these new forms of criminal behavior that are perceived as serious threats [8]. These problems can be overcome by providing novice users with information on how to deal with personal data online.

With the Internet-savvy baby boom cohort reaching older age, future research will have to shift its focus from first encounters with social media to long-term changes in social media use. (1) With the rise and fall of online social networks, long-term development of online and offline friendships and considering moves between online social networks are certainly worth investigating. (2) With growing numbers of online community members reaching advanced age, new considerations for user rights and obligations have to be taken into account. Long-term cognitive decline will pose new challenges, considering that adults with compromised decision-making ability, e.g. due to dementia, may be more susceptible to exploitation or fraud. As Batchelor et al. [25] point out, responsibility for online behavior of users with dementia cannot be upheld; meanwhile, the user's relatives are not legally allowed to modify or delete online content when they have not been authorized by the user before decisionmaking ability was compromised. Furthermore, visions of user rights enabling users to protect or even remove their online appearance still do not solve problems of compromised decision-making ability. 
To sum up, potentially negative consequences involve threats to health and well-being from adoption of potentially harmful information, harmful behaviors, and misuse of shared personal information by criminals. Management of online content and social profiles in case of compromised decision-making ability are currently not enough part of a public discussion in terms of legal and ethical considerations.

\section{Conclusions}

Social media use of older adults is an emerging topic with potential in clinical practice. Concerning prerequisites, functional capacity as well as ICT- and social mediarelated knowledge are necessary in the adoption of social media. Potentially negative consequences of social media use in older age could be to adopt harmful information via online communities and to become a victim of harmful behaviors of other users or of misuse of personal data. However, current research highlights the potential for clinical practice and positive consequences: social media participation can advance health-related knowledge on prevention, diagnosis, and treatment of conditions and disorders. In terms of meaningful social exchange, social media can be used as a means to provide and receive social support, overcome loneliness as well as to enhance feelings of control and self-efficacy.

\section{Acknowledgements}

This paper was written during a postdoctoral research fellowship funded by the National Research Fund Luxembourg under the FLARE 2 program.

\section{References}

1 Ambient Assisted Living Joint Programme. http://www.aal-europe.eu/call-2/. Accessed July 30, 2012.

2 Zickuhr K, Madden M: Older adults and Internet use. For the first time, half of adults aged 65 and older are online. http://pewinternet.org/Reports/2012/Older-adults-and-internet-use.aspx.

-3 Lee D, Helal S, Anton S, De Deugd S, Smith A: Participatory and persuasive telehealth. Gerontology 2012;58:269-281.

4 Kaplan AM, Haenlein M: Users of the world, unite! The challenges and opportunities of social media. Bus Horiz 2009;53:59-68.

5 SeniorNet Fact Sheet. http://seniornet.org/index.php?option=com_content\&task=view\& $\mathrm{id}=43 \&$ Itemid $=68$. Accessed September 13, 2012.

6 Charness N, Boot WR: Aging and information technology use. Curr Dir Psychol Sci 2009; 18:253-258.

7 Lee B, Chen Y, Hewitt L: Age differences in constraints encountered by seniors in their use of computers and the Internet. Comput Human Behav 2011;27:1231-1237.

8 Xie B, Watkins I, Golbeck J, Huang M: Understanding and changing older adults' perceptions and learning of social media. Educ Gerontol 2012;38:282.

9 Lehtinen V, Näsänen J, Sarvas R: 'A little silly and empty-headed': older adults' understandings of social networking sites. Proceedings of the 23rd British HCI Group Annual Conference on People and Computers: Celebrating People and Technology. Cambridge, British Computer Society, 2009, pp 45-54.
10 Pfeil U, Zaphiris P, Wilson S: Older adults' perceptions and experiences of online social support. Interact Comput 2009;21:159-172.

11 Nimrod G: Seniors' online communities: a quantitative content analysis. Gerontologist 2010;50:382-392.

12 Xie B: Multimodal computer-mediated communication and social support among older Chinese Internet users. J Comput Mediated Commun 2008;13:728-750.

13 Lewandowski J, Rosenberg BD, Parks MJ, Siegel JT: The effect of informal social support: face-to-face versus computer-mediated communication. Comput Human Behav 2011;27: 1806-1814.

14 Cornejo R, Favela J, Tentori M: Ambient displays for integrating older adults into social networking sites. Collaboration and technology. Lec Notes Comput Sci 2010;6257:321336.

15 Jaeger PT, Bo Xie: Developing online community accessibility guidelines for persons with disabilities and older adults. J Disabil Policy Stud 2009;20:55-63.

16 Perkins EA, LaMartin KM: The Internet as social support for older carers of adults with intellectual disabilities. J Policy Pract Intellect Disabil 2012;9:53-62.

17 Saleh J, Robinson BS, Kugler NW, Illingworth KD, Patel P, Saleh KJ: Effect of social media in health care and orthopedic surgery. Orthopedics 2012;35:294-297.
18 Barak A, Boniel-Nissim M, Suler J: Fostering empowerment in online support groups. Comput Hum Behav 2008;24:1867-1883.

19 Nahm ES, Barker B, Resnick B, Covington B, Magaziner J, Brennan PF: Effects of a social cognitive theory-based hip fracture prevention web site for older adults. Comput Inform Nurs 2010;28:371-379.

20 Wright K: Computer-mediated social support, older adults, and coping. J Commun 2000;50:100-118.

21 Tanis M, Das E, Fortgens-Sillmann M: Finding care for the caregiver? Active participation in online health forums attenuates the negative effect of caregiver strain on wellbeing. Commun Eur J Commun Res 2011;36: 51-66.

22 Shepherd CE, Aagard S: Journal writing with Web 2.0 tools: a vision for older adults. Educ Gerontol 2011;37:606-620.

23 Luxton DD, June JD, Fairall JM: Social media and suicide: a public health perspective. Am J Public Health 2012;102:S195-S200.

24 Chou HT, Edge N: 'They are happier and having better lives than I am': the impact of using Facebook on perceptions of others' lives. Cyberpsychol Behav Soc Netw 2012;15:117-121.

25 Batchelor R, Bobrowicz A, Mackenzie R, Milne A: Challenges of ethical and legal responsibilities when technologies' uses and users change: social networking sites, decisionmaking capacity and dementia. Ethics Inf Technol 2012;14:99-108. 